\title{
Levantamento preliminar da mastofauna da região de Anajás-Muaná, Ilha de Marajó, Pará, Brasil
}

\author{
Suely A. Marques-Aguiar ${ }^{1}$ \\ Cláudia C. S. Melo ${ }^{2}$ \\ Gilberto F. Souza Aguiar ${ }^{3}$ \\ João Alberto L. Queiróz ${ }^{1}$
}

\begin{abstract}
Preliminary survey of the mammalian fauna in the Anajás-Muaná region, Marajó Island, Pará State, Brazil. A short inventory of the mammalian fauna occurring in an area of the Marajó Island was performed during the rainy season. In addition to captures by using Sherman and Tomahawk traps, and mist-nets, indirect evidence was considered, e.g. finding of body fragments of specimens, vocalization, and testimonies from local people. A total of 57 species ( 22 families) was recorded. None of them is presently considered as critically endangered according to the IUCN criteria. First confirmed record for the fruit-eating bat Platyrrhinus brachycephalus (Rouk \& Carter, 1972) in the Marajó archipelago. Additional surveys conducted in both wet and dry seasons are required for improving the profile of richness and diversity of mammal species in that region.

KEY WORDS. Mammals survey, Marajó Island, Amazonia
\end{abstract}

O Brasil é considerado o país ocidental provido da maior diversidade de mamíferos do mundo (FONSECA et al. 1999). Cerca de 60\% das mais de quinhentas espécies descritas em seu território ocorrem na porção brasileira da Amazônia, muitas delas endêmicas (SILVA et al. 2001). Do ponto de vista biogeográfico, a mastofauna amazônica exibe um padrão heterogêneo para marsupiais, quirópteros, primatas e roedores, e homogêneo para edentados, carnívoros e ungulados (Voss \& EMMONS 1996).

As ilhas que compõem o arquipélago de Marajó, no estuário do Amazonas, caracterizam-se pela grande variabilidade de dimensão territorial, e importante diversidade fitofisionômica. Em suas encostas se encontram extensas praias, e em seu interior uma teia hidrográfica (rios, furos, igarapés) que abastece e entrecorta matas de terra firme, várzeas, campos e espaços ocupados pela população ribeirinha.

A ilha de Marajó, mais importante do arquipélago, e também a maior do mundo no gênero flúvio-marítimo $\left(50 \mathrm{mil} \mathrm{km}^{2}\right)$, vem sofrendo pressão antrópica de moderada magnitude, através da atividade agropastoril e extrativista. Parte de seu

1) Museu Paraense Emílio Goeldi, Coordenação de Zoologia, Mastozoologia. Caixa Postal 399, 66040-170 Belém, Pará, Brasil. E-mail: samaguiar@museu-goeldi.br

2) Museu Paraense Emílio Goeldi, Programa de Geologia Histórica. Caixa Postal 399, 66040-170 Belém, Pará, Brasil.

3) Museu Paraense Emílio Goeldi, Coordenação de Ciências Humanas. Caixa Postal 399 , 66040-170 Belém, Pará, Brasil. 
território é tida como prioritária para inventários imediatos e planos de conservação, em virtude de desmatamentos, principalmente de madeiras nobres e palmeirais (CAPOBIANCO et al. 2001).

VIVo (1996) e SiLVA JR. (1998) relatam que a baixa amostragem constitui o maior obstáculo à caracterização da diversidade mastofaunística no Brasil globalmente. É também este o caso particular da foz do Amazonas. Os inventários existentes derivam de coletas feitas em pontos isolados, dispersos em áreas amplas (ÁvILA-PIRES 1958; PICCININI 1974; REIS \& SCHUBART 1979; MOK et al. 1982) ou direcionados para algumas ordens, com abordagem biogeográfica recente de primatas que inclui ilhas menores adjacentes - Caviana, Mexiana e Gurupá (AYRES et al. 1989; PeRES 1989; Fernandes 1994; Fernandes et al. 1995).

O objetivo deste trabalho consiste em apresentar um quadro sucinto e inicial da diversidade e status de conservação da mastofauna em uma extensão do centrosudeste da ilha de Marajó.

\section{MATERIAL E MÉTODOS}

\section{Área de estudo}

Trecho de cerca de $30 \mathrm{~km}$ de extensão em linha reta entre as nascentes dos

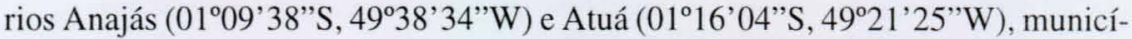
pios de Anajás (microrregião do Furo de Breves) e Muaná (microrregião do Arari), estado do Pará, Brasil (Fig. 1). Paisagens do percurso: mata de terra firme, várzea, campos e capoeira.

\section{Atividades de campo}

A expedição sucedeu-se de 19 a 29 de janeiro de 1998 (estação chuvosa), com oito dias de amostragem. No inventário se admitiram quatro categorias de registros: (a) captura; (b) achado de partes corporais; (c) registro indireto por membro da equipe (p.e. avistamento, vocalização); (d) relato da população local.

Para marsupiais, roedores e lagomorfos se utilizaram armadilhas de captura viva (20 Sherman e 10 Tomahawk) no espaço de cinco dias. As iscas consistiram de pasta de amendoim, emulsão de Scotch e porções de macaxeira e abacaxi.

Para a captura noturna de morcegos se utilizaram redes de neblina suspensas até cerca de três metros de altura do solo, atendendo-se a procedimentos-padrão para táxons neotropicais (WILSON et al. 1970; MARQUES 1985a,b; GEORGE et al. 1988; OVERAL \& MASCARENHAS 1993; AsCORRA et al. 1996; VOSS \& EMMONS 1996; WILSON et al. 1996) e considerando-se a diferenciação de paisagens. Nove redes permaneceram abertas entre 18:00 e 24:00 h nos seis primeiros dias. Nos últimos dois dias, este período foi ampliado, procedendo-se à coleta de 18:00 h de um dia às 6:00 $\mathrm{h}$ do dia seguinte. $\mathrm{O}$ trabalho diurno consistiu na exploração de abrigos, e.g. troncos de árvores e folhas de palmeiras.

Para todos os táxons se assumiu como válido o achado de partes corporais (carcaças, crânios, pós-crânios, caudas) de indivíduos mortos, naturalmente ou vítimas de caça. Consideraram-se também observações de membros da equipe e depoimentos de cerca de 30 ribeirinhos da região, especialmente sobre a presença 
de grupos aquáticos e semi-aquáticos, e aqueles com representantes de grande porte. Ocasionalmente se recorreu a arma de fogo (espingarda calibre 20, chumbo em roseta).

O método de censo por transecto de primatas se tornou impraticável em virtude das condições alagadiças e o curto período de visitação.

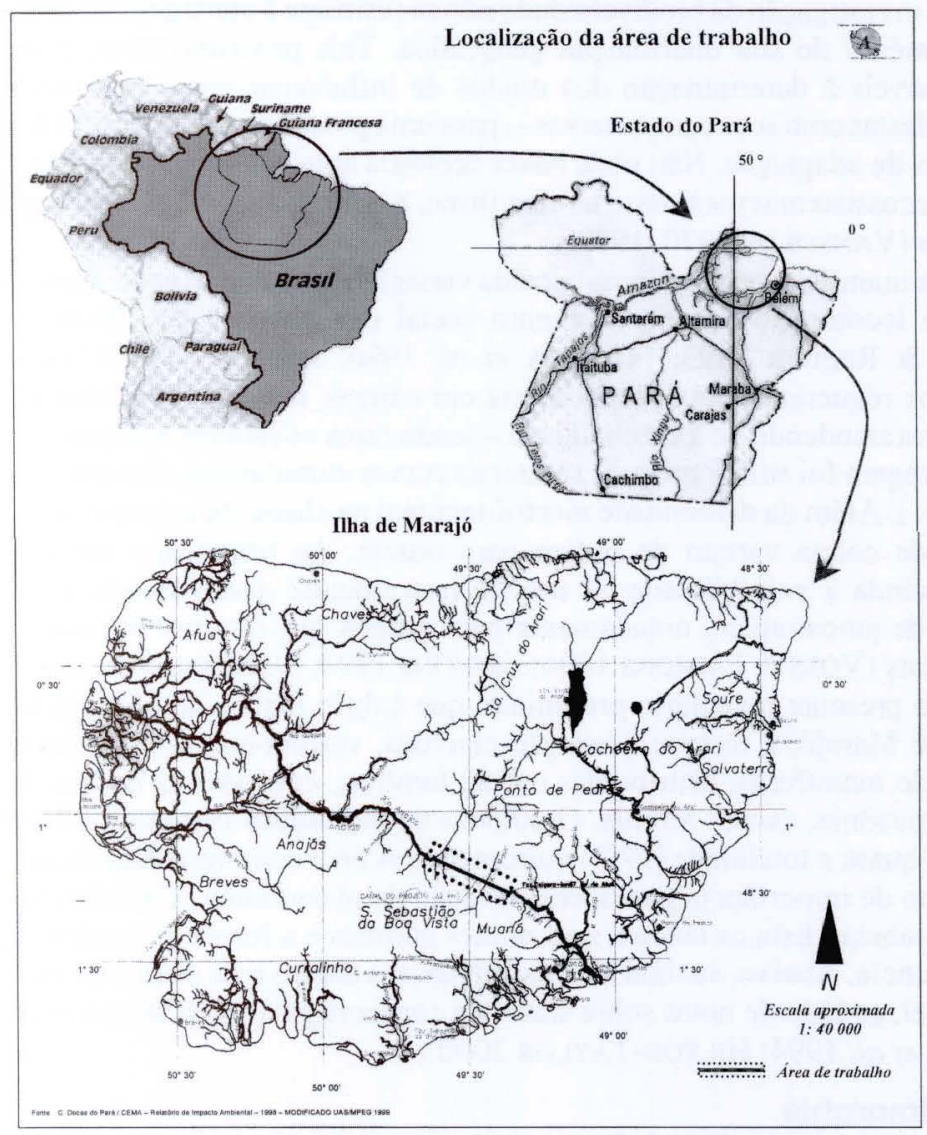

Fig. 1. Ilha de Marajó e região amostrada.

\section{Tratamento do material e curadoria}

Em torno de $95 \%$ dos exemplares coletados foram fixados em formol a $10 \%$, e conservados em álcool a $70^{\circ} \mathrm{GL}$, sendo o restante taxidermizado.

A identificação em nível de espécie se baseou em chaves classificatórias (VIZOTTO \& TADEI 1973; Jones \& CARTER 1976; HANDLEy 1987, 1990, 1991; NOWAK 1991; MARQUES-AGUIAR 1994; EMMONS 1997) e comparação com espécimes identificados da coleção mastozoológica do Museu Paraense Emílio Goeldi, Coordenação de Zoologia. 
Todo o resultado da coleta foi incorporado ao patrimônio do MPEG, coleção mastozoológica (MPEG 25056-25400) e coleção osteológica do Programa de Geologia Histórica (CO-MPEG 088-099, 101, 103, 104, 106-111 e 113).

\section{RESULTADOS E DISCUSSÃO}

A investigação da biodiversidade não se restringe à contagem de organismos e mapeamento de sua distribuição geográfica. Tais procedimentos, porém, são indispensáveis à determinação dos modos de influências entre espécies - e das relações destas com seus ecossistemas -, para uma posterior compreensão dos meios e sistemas de adaptação. Não pode haver ecologia aplicada (conservação e recuperação de ecossistemas) sem base teórica firme, a qual não se obtém sem exploração descritiva (VANZOLINI 1970, 1977).

Os mamíferos exibem pronunciada variação de tamanho, peso, dieta, hábitat, meios de locomoção e comportamento social (CABRERA 1958; NOWAK 1991; WILSON \& REEDER 1993; FONSECA et al. 1996; EMMONS 1997). Inventários detalhados requerem longa permanência em campo, mais de uma visitação - de preferência atendendo-se à sazonalidade - sendo raros os estudos nos quais o esforço de amostragem foi suficiente para saturar as curvas cumulativas de espécies (SILVA et al. 2001). Além da diversidade morfofuncional na classe, bem como de nicho, as técnicas de coleta variam de ordem para ordem. As limitações metodológicas incluem ainda a exigibilidade de uso de instrumental diversificado e múltiplos métodos de amostragem, notadamente para grupos que têm por hábitat as matas neotropicais (VOSS \& EMMONS 1996; EMMONS 1997; SimMONS \& Voss 1998).

No presente inventário preliminar, que cobriu menos de $10 \%$ do território da ilha de Marajó, e restrito à estação chuvosa, verificou-se a ocorrência de 57 espécies de mamíferos, distribuídas em 22 famílias, em todas as ordens descritas para a Amazônia, exceto Sirenia. O número de indivíduos capturados foi de 327, dos quais quase a totalidade (97\%) quirópteros. A área compreendida inclui pontos tidos como de importância para a conservação da mastofauna (CAPOBIANCO et al. 2001). A tabela I lista os táxons, seus nomes comuns e a forma de evidenciação de sua ocorrência. Abaixo, sucinta abordagem desses táxons e de outros de ocorrência presumível, ao lado de notas sobre status de conservação (BERNARDES et al. 1990; FONSECA et al. 1994; HILTON-TAYLOR 2000).

\section{Didelphimorphia}

A maioria dos marsupiais neotropicais possui hábitos noturnos e suas populações costumam sofrer drásticas flutuações de densidade, o que dificulta sua coleta e até mesmo seu simples avistamento (Voss \& EMMONS 1996). Houve registro direto de uma espécie (Didelphis marsupialis Linnaeus, 1758, mucura, gambá) e indireto de duas outras (Chironectes minimus (Zimmermann, 1780), mucura d'água, e Philander opossum (Linnaeus, 1758), mucura quatro-olhos). Dotado de cauda prêensil, D. marsupialis pode locomover-se em meio a partes arbóreas altas, guardando distância do solo. A equipe capturou um macho e uma fêmea com três filhotes. C. minimus foi apenas avistado, sem captura. EMMONS (1997) relata baixa densidade desta espécie em grande parte de sua distribuição. Moradores referiram $P$. opossum, mas também em baixa densidade. 
Tabela I. Taxons de mamíferos com registros. (1) Número de indivíduos capturados (para quirópteros, número de machos entre parênteses); (2) número de fragmentos corporais encontrados no percurso; (3) observação por membro da equipe, ou registro de vocalização; (4) relato de morador/caçador.

\begin{tabular}{|c|c|c|c|c|c|}
\hline Táxons & Nomes comuns & 1 & 2 & 3 & 4 \\
\hline \multicolumn{6}{|l|}{ Didelphimorphia } \\
\hline \multicolumn{6}{|l|}{ Didelphidae } \\
\hline Chironectes minimus (Zimmermann, 1780) & Mucura d'água & - & - & $\mathrm{x}$ & - \\
\hline Didelphis marsupialis Linnaeus, 1758 & Mucura, gambá & 5 & - & - & - \\
\hline Philander opossum (Linnaeus, 1758) & Mucura quatro-olhos & - & - & - & $\mathrm{x}$ \\
\hline \multicolumn{6}{|l|}{ Xenarthra } \\
\hline \multicolumn{6}{|l|}{ Bradypodidae } \\
\hline Bradypus variegatus Schinz, 1825 & Preguiça-bentinha & 1 & 1 & - & - \\
\hline \multicolumn{6}{|l|}{ Mirmecophagidae } \\
\hline Cyclopes didactylus (Linnaeus, 1758) & Tamanduaí & - & - & - & $x$ \\
\hline Tamandua tetradactyla (Linnaeus, 1758) & Mambira, tamanduá-de-colete & - & - & - & $x$ \\
\hline \multicolumn{6}{|l|}{ Dasypodidae } \\
\hline Cabassous unicinctus (Linnaeus, 1758) & Tatu rabo-de-couro & - & - & - & $\mathrm{x}$ \\
\hline Dasypus septemcinctus Linnaeus, 1758 & Tatui & - & - & - & $\mathrm{x}$ \\
\hline Dasypus novemcinctus Linnaeus, 1758 & Tatu-galinha & - & 13 & - & - \\
\hline \multicolumn{6}{|l|}{ Chiroptera } \\
\hline \multicolumn{6}{|l|}{ Noctilionidae } \\
\hline Noctilio leporinus (Linnaeus, 1758) & Morcego pescador & $3(3)$ & - & - & - \\
\hline \multicolumn{6}{|l|}{ Phyllostomidae } \\
\hline Trachops cirrhosus (Spix, 1823) & Morcego & 1 & - & - & - \\
\hline Choeroniscus minor (Peters, 1868) & Morcego & 1 & - & - & - \\
\hline Glossophaga soricina (Pallas, 1766) & Morcego & $3(3)$ & - & - & - \\
\hline Lonchophylla thomasi J.A. Allen, 1904 & Morcego & 1 & - & - & - \\
\hline Carollia perspicillata (Linnaeus, 1758) & Morcego & $66(19)$ & - & - & - \\
\hline Rhinophylla pumilio Peters, 1865 & Morcego & $4(1)$ & - & - & - \\
\hline Ametrida centurio Gray, 1847 & Morcego & $6(6)$ & - & - & - \\
\hline Artibeus cinereus (Gervais, 1856) & Morcego & $22(12)$ & - & - & - \\
\hline Artibeus concolor Peters, 1865 & Morcego & $2(1)$ & - & - & - \\
\hline Artibeus gnomus Handley, 1987 & Morcego & 2 & - & - & - \\
\hline Artibeus jamaicensis Leach, 1821 & Morcego & $32(12)$ & - & - & - \\
\hline Artibeus lituratus (Olfers, 1818) & Morcego & $11(5)$ & - & - & - \\
\hline Artibeus obscurus Schinz, 1821 & Morcego & $84(38)$ & - & - & - \\
\hline Artibeus sp. & Morcego & $7(4)$ & - & - & - \\
\hline Platyrihinus brachycephalus (Rouk \& Carter, 1972) & Morcego & $1(1)$ & - & - & - \\
\hline Platyrrhinus helleri (Peters, 1866) & Morcego & $7(2)$ & - & - & - \\
\hline Sturnira lilium (E. Geoffroy, 1810) & Morcego & $14(4)$ & - & - & - \\
\hline Sturnira tildae de la Torre, 1959 & Morcego & $2(1)$ & - & - & - \\
\hline Uroderma bilobatum Peters, 1866 & Morcego & $24(5)$ & - & - & - \\
\hline Uroderma magnirostrum Davis, 1968 & Morcego & 1 & - & - & - \\
\hline Vampyressa bidens (Dobson, 1878) & Morcego & 1 & - & - & - \\
\hline Desmodus rotundus (E. Geoffroy, 1810) & Morcego vampiro & $23(15)$ & - & - & - \\
\hline \multicolumn{6}{|l|}{ Primates } \\
\hline \multicolumn{6}{|l|}{ Callitrichidae } \\
\hline Saguinus midas (Linnaeus, 1758) & Sagüi, sauim & 1 & - & - & - \\
\hline \multicolumn{6}{|l|}{ Cebidae } \\
\hline Alouatta belzebul (Linnaeus, 1766) & Guariba & - & - & $x$ & $\mathrm{x}$ \\
\hline Aotus infulatus (Kuhl, 1920) & Macaco-da-noite & - & - & - & $x$ \\
\hline Cebus apella (Linnaeus, 1758) & Macaco-prego & - & 2 & $x$ & - \\
\hline Saimiri sciureus (Linnaeus, 1758) & Macaco-de-cheiro & - & - & $x$ & - \\
\hline \multicolumn{6}{|l|}{ Carnivora } \\
\hline \multicolumn{6}{|l|}{ Felidae } \\
\hline Leopardus pardalis (Linnaeus, 1758) & Jaguatirica & - & 2 & $x$ & - \\
\hline Puma concolor (Linnaeus, 1771) & Suçuarana & - & - & - & $x$ \\
\hline Panthera onca (Linnaeus, 1758) & Onça pintada & - & - & - & $x$ \\
\hline \multicolumn{6}{|l|}{ Mustelidae } \\
\hline Eira barbara (Linnaeus, 1758) & Irara & - & - & $x$ & - \\
\hline Lontra longicaudis (Olfers, 1818) & Lontra & - & - & $x$ & - \\
\hline \multicolumn{6}{|l|}{ Procyonidae } \\
\hline Nasua nasua (Linnaeus, 1766) & Quati & - & 4 & - & - \\
\hline Procyon cancrivorus (G. Cuvier, 1798) & Guaxinim & - & 1 & - & - \\
\hline
\end{tabular}


Tabela I. Continuação.

\begin{tabular}{|c|c|c|c|c|c|}
\hline Táxons & Nomes comuns & 1 & 2 & 3 & 4 \\
\hline \multicolumn{6}{|l|}{ Cetacea } \\
\hline $\begin{array}{l}\text { Sotalia fluviatilis (Gervais \& Deville, 1853) } \\
\text { Perissodactyla }\end{array}$ & Boto-tucuxi & - & - & $x$ & $x$ \\
\hline \multicolumn{6}{|l|}{ Tapiridae } \\
\hline \multicolumn{6}{|l|}{ Artiodactyla } \\
\hline \multicolumn{6}{|l|}{ Tayassuidae } \\
\hline Pecari tajacu (Linnaeus, 1758) & Caititu & - & 4 & - & - \\
\hline Tayassu pecari (Link, 1795) & Queixada & - & - & - & $\mathrm{x}$ \\
\hline \multicolumn{6}{|l|}{ Cervidae } \\
\hline Mazama americana (Erxleben, 1777) & Veado-vermelho & - & 5 & - & - \\
\hline \multicolumn{6}{|l|}{ Rodentia } \\
\hline \multicolumn{6}{|l|}{ Sciuridae } \\
\hline $\begin{array}{l}\text { Sciurus aestuans Linnaeus, } 1766 \\
\text { Erethizontidae }\end{array}$ & Esquilo, quatipuru & 1 & - & - & - \\
\hline Coendou prehensilis (Linnaeus, 1758) & Coendu & - & - & - & $\mathrm{x}$ \\
\hline \multicolumn{6}{|l|}{ Dasyproctidae } \\
\hline Dasyprocta fuliginosa Wagler, 1832 & Cotia cinza & - & - & - & $\mathrm{x}$ \\
\hline Dasyprocta leporina (Linnaeus, 1758) & Cotia vermelha & - & - & $x$ & - \\
\hline \multicolumn{6}{|l|}{ Agoutidae } \\
\hline \multicolumn{5}{|l|}{ Hydrochaeridae } & - \\
\hline $\begin{array}{l}\text { Hydrochaeris hydrochaeris (Linnaeus, 1766) } \\
\text { Echimyidae }\end{array}$ & Capivara & - & - & - & $x$ \\
\hline \multicolumn{5}{|l|}{ Lagomorpha } & - \\
\hline Leporidae & & & & & \\
\hline Sylvilagus brasiliensis (Linnaeus, 1758) & Coelho comum & - & - & - & $\mathrm{x}$ \\
\hline
\end{tabular}

Possível ocorrência de Caluromys lanatus (Olfers, 1818) (mucura-chichica ou cuíca) e dos gêneros Gracilinanus Gardner \& Creighton, 1989, Marmosa Gray, 1821, Marmosops Matschie, 1916, Metachirus Burmeister, 1854, Micoureus Lesson, 1842 e Monodelphis Burnett, 1830 (NOWAK 1991; MASCARENHAS et al. 1992; MirandA-NeTO 1993; Voss \& EMMONS 1996; EMMONS 1997; EISENBERG \& REDFORD 1999).

\section{Xenarthra}

Registro de uma espécie de preguiça, duas de tamanduá e três de tatu.

As preguiças recentes, arbóreas, basicamente só descem ao solo para defecação, fator que muito dificulta sua amostragem. Capturou-se um exemplar de Bradypus variegatus Schinz, 1825 (preguiça-bentinha) e se recolheu a ossatura craniana de um segundo exemplar.

VOSS \& EMMONS (1996) destacam a dificuldade também na captura de mirmecofagídeos usando-se armadilhas convencionais. Duas espécies foram referidas por moradores: Cyclopes didactylus (Linnaeus, 1758) (tamanduaí) e Tamandua tetradactyla (Linnaeus, 1758) (mambira, tamanduá-de-colete).

A existência de tatus na região pode ser constatada de modo indireto, localizando-se tocas, mas estas, que são conspícuas na estiagem, praticamente desaparecem quando se inicia a estação chuvosa. No percurso recolhemos caudas de onze indivíduos e dois fragmentos esqueletais cranianos de Dasypus novemcinctus Linnaeus, 1758 (tatu-galinha). Presença relatada de Cabassous unicinctus (Linnaeus, 1758) (tatu-rabo-de-couro) e D. septemcinctus Linnaeus, 1758 (tatuí). 
Outras espécies, sugeridas na literatura: Bradypus tridactylus Linnaeus, 1758 (também denominada preguiça-bentinha), Myrmecophaga tridactyla Linnaeus, 1758 (tamanduá-bandeira, vulnerável), Priodontes maximus (Kerr, 1792) (tatucanastra, em perigo de extinção), Choloepus didactylus (Linnaeus, 1758) (preguiça-real), Dasypus kappleri Krauss, 1862 (tatu-canastra, homônimo de $P$. maximus) e Euphractus sexcinctus (Linnaeus, 1758) (tatu-peludo, tatupeba) (NowAK 1991; MASCARENHAS et al. 1992; MIRANDA-NETO 1993; VOSS \& EMMONS 1996; EMMONS 1997; EISENBERG \& REDFORD 1999).

\section{Chiroptera}

A elevada diversidade de dieta dos morcegos neotropicais os torna importantes na preservação do equilíbrio dos ecossitemas onde ocorrem. Atuam no controle populacional da entomofauna, dispersão de sementes, polinização e reservatório do vírus rábico (GRASSÉ 1955; PIJL 1957; VOGEL 1969; SLAUGHTER \& WALTON 1970; TADDEI 1983; WILSON 1989; NOWAK 1991, 1994; WILSON 1997; MARINHO-FILHO \& SAZIMA 1998). A formação de áreas antrópicas é acompanhada de rarefação de certos grupos e concentração de outros originalmente menos representativos (MARQUES 1989). Tal susceptibilidade a variações de diversidade serve de base ao emprego de quirópteros como bioindicadores de perturbação ambiental (JoHns et al. 1985; MARQUES-AGUIAR 1994; WILSON et al. 1996).

A ordem revelou-se predominante na região de Anajás-Atuá. Ao longo do trecho explorado se observaram 23 espécies, todas, à exceção de Noctilio leporinus (Linnaeus, 1758) (morcego-pescador), pertencentes à família Phyllostomidae, a maioria com hábito de frugivoria, nenhuma insetívora preferencial. No gênero Artibeus Leach, 1821, com mais da metade da coleta, seis espécies: A. cinereus (Gervais, 1856), A. concolor Peters, 1865, A. gnomus Handley, 1987, A. jamaicensis Leach, 1821, A. lituratus (Olfers, 1818) e A. obscurus Schinz, 1821 - as três últimas frugívoras oportunistas, compondo $40 \%$ da amostra na ordem. A elevada representatividade do gênero, como também de Carollia perspicillata (Linnaeus, 1758), constitui indício de mudanças de paisagem pelo impacto humano. Importantes dispersores de sementes, estes grupos contribuem no processo de regeneração florestal (HANDLEY et al. 1991; Cloutier \& ThOMAS 1992; MARQues-AguiAR 1994; EMmOns 1997). Outras espécies neotropicais predominantemente frugívoras amostradas: Rhinophylla pumilio Peters, 1865, Sturnira lilium (E. Geoffroy, 1810), S. tildae de la Torre, 1959, Ametrida centurio Gray, 1847 (pouco estudado e de difícil captura), Platyrrhinus brachycephalus (Rouk \& Carter, 1972) (baixa incidência na Amazônia, primeiro registro confirmado na ilha), $P$. helleri (Peters, 1866), Uroderma bilobatum Peters, 1866, $U$. magnirostrum Davis, 1968 e Vampyressa bidens (Dobson, 1878). Este predomínio de frugívoros possivelmente decorre de quatro fatores: (a) dificuldades de captura de insetívoros com redes de neblina, em virtude de um sistema de radar especialmente acurado que permite fácil detecção dessas redes; (b) há algumas espécies, inclusive insetívoras, que ocorrem mais comumente no dossel arbóreo (acima de $30 \mathrm{~m}$ do solo, HANDLEY 1967), o que conduz a uma subestimativa da incidência e distribuição em avaliações rápidas preliminares; (c) amostragem única na estação chuvosa; (d) limitações de permanência em campo e mascaramento dos efeitos de sazonalidade, especialmente ciclos lunares. 
Entre os morcegos de hábitos nectarívoros: Choeroniscus minor (Peters, 1868), Glossophaga soricina (Pallas, 1766) e Lonchophylla thomasi J.A. Allen, 1904. Coletou-se uma fêmea adulta de Trachops cirrhosus (Spix, 1823), espécie que preda rãs e lacertílios, ocasionalmente aves e mamíferos de pequeno porte. A presença de $N$. leporinus, amplamente distribuído mas não de fácil captura, sugere riqueza da ictiofauna.

De reforço à hipótese de mudanças causadas pela ocupação humana tem-se o achado de Desmodus rotundus (E. Geoffroy, 1810) (morcego vampiro, 23 animais amostrados), de grande interesse epidemiológico pela hematofagia e eventual disseminação do vírus rábico. Ataca mamíferos, excepcionalmente aves. Normalmente raro em mata primária, torna-se comum em ambientes antrópicos, tais como áreas de pasto (EMMONS 1997).

Este perfil preliminar de riqueza e diversidade é sugestivo da presença de mais de 50 espécies de morcegos globalmente na ilha de Marajó, em gêneros de insetívoros (Rhynchonycteris Peters, 1867, Saccopteryx Illiger, 1811, Peropteryx Peters, 1867, Pteronotus Gray, 1838, Macrophyllum Gray, 1838, Furipterus Bonaparte, 1837, Thyroptera Spix, 1823, Myotis Kaup, 1829, Eptesicus Rafinesque, 1820, Eumops Miller, 1906 e Molossus E. Geoffroy, 1805), frugívoros (Chiroderma Peters, 1860, Mesophylla Thomas, 1901 e Vampyrodes Thomas, 1900), nectarívoros (Lionycteris Thomas, 1913) e onívoro-carnívoros (Chrotopterus Peters, 1865 e Phyllostomus Lacépède, 1799) (NOWAK 1994; Voss \& EMMONS 1996; EMMONS 1997; EISENBERG \& REDFORD 1999).

\section{Primates}

Tanto cebídeos quanto calitriquídeos possuem representantes no arquipélago de Marajó (FERNANDES et al. 1995). Na área de amostragem, registro de cinco espécies, corroborando as coleções de referência do MPEG e do MNRJ (Museu Nacional, Rio de Janeiro): Alouatta belzebul (Linnaeus, 1766) (guariba), Cebus apella (Linnaeus, 1758) (macaco-prego), Saimiri sciureus (Linnaeus, 1758) (macaco-de-cheiro), Aotus infulatus (Kuhl, 1920) (macaco-da-noite) e Saguinus midas (Linnaeus, 1758) (sagüi, sauim). A equipe capturou apenas um indivíduo de $S$. midas, abatido com arma de fogo. Trata-se de espécie domesticável que pode ter sido introduzida com o influxo humano. Presença da subespécie $S$. midas niger já referida por FERNANDES et al. (1995) mas sem relato para as demais ilhas maiores (Caviana, Mexiana e Gurupá). Maior primata encontrado no Brasil, distribuído em várias regiões do país mas quase extinto em estados do Nordeste, $A$. belzebul teve sua ocorrência constatada pelo reconhecimento da emissão vocal característica de espécimes nas imediações dos pontos de amostragem. Depoimento de ribeirinhos reforçou o achado, já anteriormente estabelecido por FERNANDES (1994). Um indivíduo de Cebus apella foi avistado pela equipe, e recolhidos dois fragmentos de crânio. A. infulatus foi relatado por moradores e $S$. sciureus avistado. As subespécies $A$. belzebul ululata e $C$. apella robustus, que constam entre as 29 referências de mamíferos brasileiros sob perigo de extinção segundo a IUCN (HILTON-TAYLOR 2000), são de improvável ocorrência no arquipélago marajoara MiRANDA-NETO (1993) e EISENBERG \& REDFORD (1999) sugerem outros táxons: 
Chiropotes satanas (Hoffmannsegg, 1807) (cuxiú, subespécie C. satanas satanas em perigo de extinção), Mico argentatus (Linnaeus, 1766) e representantes dos gêneros Ateles É. Geoffroy, 1806 (coatá), Callicebus Thomas, 1903 (zogue-zogue) e Lagothrix É. Geoffroy, 1812 (barrigudo).

\section{Carnivora (exceto mustelídeos)}

Os hábitos predominantemente noturnos e a baixa densidade populacional torna exigível maior permanência em campo para um inventário detalhado dos carnívoros neotropicais - pelo menos alguns meses. Indicada a ocorrência na região de duas espécies de procionídeos (Nasua nasua (Linnaeus, 1766), quati, e Procyon cancrivorus (G. Cuvier, 1798), guaxinim) e três de felídeos (Leopardus pardalis (Linnaeus, 1758), jaguatirica, Puma concolor (Linnaeus, 1771), suçuarana, e Panthera onca (Linnaeus, 1758), onça-pintada).

A equipe encontrou quatro ossaturas cranianas sem mandíbula de $N$. nasua e um crânio de $P$. cancrivorus. Apesar da extensa distribuição de ambos, são restritos os dados sobre sua diversidade nos neotrópicos. Diferentemente dos felídeos, que são exclusivamente carnívoros e predadores de vertebrados (de peixes a outros mamíferos de médio porte), os procionídeos alimentam-se predominantemente de invertebrados (moluscos, crustáceos) ou pequenos vertebrados (peixes, anfíbios), excepcionalmente até partes vegetais.

Não se atestou a existência local de felídeos mas esta é sugerida por ribeirinhos, tendo havido registro de $L$. pardalis pelo achado de um crânio e um pós-crânio. EMMONS (1997) não inclui a ilha de Marajó no mapeamento de distribuição de $P$. onca, mas EISENBERG \& REDFORD (1999) a incluem, inclusive ao lado de Leopardus tigrinus (Schreber, 1774) (gato-do-mato). A população local refere a ocorrência da espécie, assim como de $P$. concolor. Apesar de muito visados como caça, especialmente para a extração e tráfico de suas peles, não integram a lista da IUCN de mamíferos ameaçados. Outras espécies esperadas: Potos flavus (Schreber, 1774) (jupará), Herpailurus yaguarondi (Lacépède, 1809) (gato-mourisco ou maracajá-preto), Leopardus wiedii (Schinz, 1821) (gato-do-mato ou maracajá-peludo), Speothos venaticus Lund, 1842 (cachorro-do-mato, vulnerável), Atelocynus microtis (Sclater, 1883) (cachorro-do-mato de orelha curta) e Cerdocyon thous (Linnaeus, 1766) (graxaim, raposa) (BERNARDES et al. 1990; MIRANDA-NETO 1993; FONSECA et al. 1994; EMMONS 1997; EISENBERG \& REDFORD 1999; SILVA JR. \& SOARES 1999).

\section{Mustelídeos e táxons aquáticos}

Duas espécies de carnívoros da família Mustelidae (Eira barbara (Linnaeus, 1758), irara, e Lontra longicaudis (Olfers, 1818), lontra) e uma espécie de Cetacea (Sotalia fluviatilis (Gervais \& Deville, 1853), boto-tucuxi).

Carnívoro preferencial e de larga distribuição, E. barbara é comumente vista em áreas perturbadas e proximidades de assentamentos humanos, enquanto $L$. longicaudis é vítima de caçadores que a abatem para o tráfico de peles (BERNARDES et al. 1990; MASCARENHAS et al. 1992; FONSECA et al. 1994). De hábitos semi-aquáticos, ambas evidenciadas por testemunho de membros da equipe. A primeira não 
consta na lista de mamíferos brasileiros ameaçados e a segunda se encontra sob estudo quanto a seu status de conservação (HILTON-TAYLOR 2000).

A presença de $S$. fluviatilis, espécie inteiramente aquática adaptada tanto para águas doces como salgadas, foi evidenciada pelo reconhecimento de ondas de vocalização e relato de morador.

A única espécie de Sirenia existente na Bacia Amazônica - Trichechus inunguis (Natterer, 1883) (peixe-boi), status de conservação vulnerável - não teve seu registro indicado para a região de Anajás-Muaná.

Ocorrência possível de Mustela africana Desmarest, 1818 (doninha), Pteronura brasiliensis (Gmelin, 1788) (ariranha, em perigo de extinção), Galictis vittata (Schreber, 1776) (furão) e Inia geoffrensis (de Blainville, 1817) (boto vermelho, vulnerável) (MIRANDA-NETO 1993; EMMONS 1997; EISENBERG \& REDFORD 1999; HILTON-TAYLOR 2000).

\section{Perissodactyla e Artiodactyla}

Quatro espécies: Tapirus terrestris (Linnaeus, 1758) (anta), Mazama americana (Erxleben, 1777) (veado vermelho), Pecari tajacu (Linnaeus, 1758) (caititu) e Tayassu pecari (Link, 1795) (queixada). Voss \& EMMONS (1996) sugerem que só ocasionalmente se podem encontrar ungulados em matas neotropicais. T. terrestris e $M$. americana, de hábitat florestal, são diurnos e noturnos, enquanto $P$. tajacu e $T$. pecari exclusivamente diurnos.

Obtidas quatro partes corporais de $P$. tajacu (três crânios e uma cauda) e cinco de M. americana (dois crânios, dois fragmentos cranianos e uma pele curtida). $T$. terrestris foi avistada pela equipe e a presença de $T$. pecari informada por morador.

Além de $M$. americana, maior veado de chifre simples, é provável a ocorrência também de $M$. gouazoubira (G. Fischer, 1814) (veado-birá ou veado catingueiro) (NOWAK 1991; MASCARENHAS et al. 1992; Voss \& EMMONS 1996; EMMONS 1997; EISENBERG \& REDFORD 1999).

\section{Rodentia e Lagomorpha}

Sete espécies de Rodentia e uma de Lagomorpha. Apenas dois indivíduos capturados (Sciurus aestuans Linnaeus, 1766, esquilo, quatipuru; e Proechimys sp., rato soiá), nenhum deles com armadilha, por razões similares às descritas para marsupiais.

Dentre as espécies com base alimentar de frutos e castanhas se destacam, ao lado de $S$. aestuans, Dasyprocta fuliginosa Wagler, 1832 (cotia cinza, primeiro registro provável na ilha, relato de morador) e Dasyprocta leporina (Linnaeus, 1758) (cotia vermelha, observação por membro da equipe).

Registro de Coendou prehensilis (Linnaeus, 1758) (coendu), Hydrochaeris hydrochaeris (Linnaeus, 1766) (capivara), relatadas por moradores, e Agouti paca (Linnaeus, 1766) (paca) pelo achado de cinco crânios. Hydrochaeris hydrochaeris e $A$. paca são perseguidas como objeto de caça por sua carne saborosa, mas não se encontram ameaçadas. Também relatada a ocorrência de Sylvilagus brasiliensis (Linnaeus, 1758) (coelho comum). 
Ao lado de marsupiais e quirópteros, os roedores representam cerca de $70 \%$ dos táxons de mamíferos na Amazônia (SILVA et al. 2001). É admissível presumir-se a ocorrência de algumas dezenas de espécies da ordem, incluindo-se nos gêneros Oryzomys Baird, 1858, Oligoryzomys Bangs, 1900, Oecomys Thomas, 1906, Nectomys Peters, 1861, Rhipidomys Tschudi, 1844 e Holochilus Brandt, 1835 (NowaK 1991; VOSS \& EMMONS 1996; EMMONS 1997; EISENBERG \& REDFORD 1999).

\section{CONCLUSÕES}

Vários fatores impuseram dificuldades ao inventário. As capturas foram prejudicadas pela elevada pluviosidade à época da expedição e restrição nas possibilidades de permanência em campo. Fez-se visitação a áreas com diversidade de paisagens, mas os espaços alagados tornaram impraticável a sistematização das coletas e estimativas de censo por transecto.

As 57 espécies aqui relatadas correspondem a cerca de $18 \%$ do total de espécies em toda a Amazônia brasileira (SILVA et al. 2001). No entanto, parece razoável assumir que a riqueza e diversidade de mamíferos no arquipélago corresponda aproximadamente à metade do total continental, em consonância com as observações de FERNANDES et al. (1995) para primatas.

É essencial que as áreas ocupadas pelos diferentes grupos estejam representadas nas coleções mastozoológicas disponíveis, pois modelos de diferenciação micro e macroevolutiva, bem como propostas de política ambiental, só poderão ser formulados quando houver uma adequada caracterização biogeográfica. Recomendam-se levantamentos mais intensivos em pontos de coleta homogeneamente distribuídos, e considerando-se variações sazonais.

AGRADECIMENTOS. À CEMA (Consultoria em Meio Ambiente, São Paulo) e MPEG, pelo apoio financeiro. Ao IBAMA, pela autorização de coleta. Ao técnico José Nílton Santa Brígida pela importante colaboração nas atividades de campo. Aos ribeirinhos da região de AnajásMuaná que gentilmente nos forneceram valiosas informações.

\section{REFERÊNCIAS}

ASCORRA, C.F.; S. Solari \& D.E. Wilson. 1996. Diversidad y ecología de los quirópteros en Pakitza, p. 593-612. In: D.E. WiLson \& A. SAndoval (Eds). Manu: The Biodiversity of Southeastern Peru. Washington, D.C., Smithsonian Institution Press, 679p.

Ávila-Pires, F.D. DE. 1958. Mamíferos colecionados nos arredores de Belém do Pará. Bol. Mus.

Paraense Emílio Goeldi, sér. Zool., 19: 1-9.

AYres, J.M.; J.I. BONSIEPE \& T.J. TOWNSHEND. 1989. Notes on monkeys and habitat in the northeast of

Marajó Island, Brazil. Primate Conservation, Washington, D.C., 10:21-22.

Bernardes, A.T.; A.B.M. Machado \& A.B. Rylands. 1990. Fauna Brasileira Ameaçada de Extinção (Brazilian Fauna Threatened with Extinction). Belo Horizonte, Fundação Biodiversitas, 62p.

Cabrera, A. 1958. Catálogo de los mamíferos de América del Sur. Rev. Mus. Argent. Cienc. Nat. Bernardino Rivadavia, Zool., 4 (1): 1-308.

Capobianco, J.P.R.; A. Veríssimo; A. Moreira; D. Sawyer; I. dos Santos \& L.P. Pinto. 2001. Biodiversidade na Amazônia brasileira. São Paulo, Estação Liberdade, Instituto Socioambiental, 540p. 
Cloutier, D \& D.W. Thomas. 1992. Carollia perspicillata. Mammalian Species 417: 1-9.

EisenberG, J.F. \& K.H. REDFord. 1999. Mammals of the Neotropics - The Central Neotropics:

Ecuador, Peru, Bolivia, Brazil. Chicago, Univ. Chicago Press, Vol. 3, 609p.

Emmons, L.H. 1997. Neotropical Rainforest Mammals: A Field Guide. Chicago, Univ. Chicago Press, $307 \mathrm{p}$.

FERnANDES, M.E.B. 1994. Notes on the Geographic Distribution of Howliong Monkeys in the Marajó Archipelago, Pará, Brazil. Int. Jour. Primatol., New York, 15 (6): 919-926.

FERnAndes, M.E.B.; J.M.C. Silva \& J.S. Silva JR. 1995. The monkey of the islands of the Amazon estuary, Brazil: a biogeographic analysis. Mammalia, Paris, 59 (2): 213-221

Fonseca, G.A.B.; G. Herrmann \& Y.L.R. Leite. 1996. Lista anotada dos mamíferos do Brasil. Conservation International \& Fundação Biodiversitas. Occ. Papers Conserv. Biol. 4: 1-38.

FonseCA, G.A.B.; G. HERRMANN \& Y.L.R. Leite. 1999. Macrogeography of Brazilian Mammals, p. 549-563. In: J.F. EISENBERG \& K.H. REDFORD (Eds). Mammals of the Neotropics. The Central Neotropics: Ecuador, Peru, Bolivia, Brazil. Chicago, Univ. Chicago Press, Vol. 3, 609p.

Fonseca, G.A.B.; A.B. Rylands; C.M.R. Costa; R.B. Machado \& Y.L.R. Leite. 1994. Livro vermelho dos mamíferos brasileiros ameaçados de extinção. Belo Horizonte, Fundação Biodiversitas, $459 \mathrm{p}$.

George, T.K.; S.A. Marques; M. De Vivo; L.C. Branch; N. Gomes \& R. Rodrigues. 1988. Levantamento de mamíferos do Parque Nacional da Amazônia (Tapajós). Brasil Florestal, Brasília, 63: $33-41$

Grassé, P.P. 1955. Mammifères. Les Ordres: Anatomie, Éthologie, Systématique. Traité de Zoologie, Paris: Masson et Cie., Vol. 17, p. 1-2300.

HANDLEY JR., C.O. 1967. Bats of the canopy of an Amazonian forest. Atas do Simpósio sobre a Biota Amazônica 5: 211-215.

1987. New species of mammals from northern South America: fruit-eating bats, genus Artibeus Leach. In: B.D. PATterson \& R.M. Timm (Eds). Studies in Neotropical mammalogy, essays in honor of Philip Herskovitz. Fieldiana Zool., N.S., Chicago, 39: 163-172.

- 1990. The Artibeus of Gray 1838, p. 443-468. In: J.F. EISEnBerg (Ed). Advances in Neotropical Mammalogy. Gainesville, Sandhill Crane Press, 614p.

. 1991. The identity of Phyllostoma planirostre Spix, 1823 (Chiroptera: Stenodermatinae). In: T.A. Griffiths \& D. KLingener (Eds). Contributions to Mammalogy in honor of Karl F. Koopman, 12-17. Bull. Amer. Mus. Nat. Hist. 206: 1-432.

Handley JR., C.O.; D.E. Wilson \& A.L. Gardner (Eds). 1991. Demography and natural history of the common fruit bat, Artibeus jamaicensis, on Barro Colorado Island, Panamá. Smith. Inst. Contrib. Zool. 511: 1-173.

Hilton-Taylor, C. 2000. The 2000 IUCN Red List of Threatened Species. Gland, Switzerland, IUCN, 61p.

JoHNS, A.D.; R.H. PINE \& D.E. WILSON. 1985. Rain forest bats - an uncertain future. Bat News, London, 5: $4-5$.

JONES JR., J.K. \& D.C. CARTER. 1976. Annotated checklist, with keys to subfamilies and genera. In: R.J. BAKER; J.K. Jones JR. \& D.C. CARTER (Eds). Biology of bats of the New World family Phyllostomatidae. Part I. Lubbock, Texas Tech Univ. Press, Special Publications, The Museum 10: 7-38.

Marinho-Filho, J. \& I. Sazima. 1998. Brazilian Bats and Conservation Biology - a first survey, p. 282-294. In: T.H. Kunz \& P.A. RACEY (Eds). Bat Biology and Conservation. Washington, D.C., Smithsonian Inst. Press, 365p.

MARques, S.A. 1985a. Novos registros de morcegos do Parque Nacional da Amazônia (Tapajós) com observações do período de atividade noturna e reprodução. Bol. Mus. Paraense Emílio Goeldi, Belém, 2 (1): 71-83.

1985b. Espécies associadas e algumas características físicas influindo na presença de Carollia perspicillata em bueiros na região de Manaus (Mammalia, Chiroptera, Phyllostomidae). 
Acta Amazonica, Manaus, 15 (1-2): 243-248.

1989. Ecologia animal: levantamento faunístico da área sob influência da BR-364

(Cuiabá-Porto Velho). Brasília, Assessoria Editorial e Divulgação Científica, 50p.

MARques-Aguiar, S.A. 1994. A systematic review of the large species of Artibeus Leach, 1821

(Mammalia: Chiroptera) with some phylogenetic inferences. Bol. Mus. Paraense Emílio Goeldi,

ser. Zool., Belém, 10 (1): 3-83.

Mascarenhas, B.M.; M.F.C. Lima \& W.L. Overal. 1992. Animais da Amazônia: guia zoológico do

Museu Paraense Emílio Goeldi. Belém, Museu Paraense Emílio Goeldi, 113p.

MoK, W.Y.; D.E. Wilson; L.A. LACEY \& R.C.C. LuizÃo. 1982. Lista atualizada de quirópteros da

Amazônia brasileira. Acta Amazônica, Manaus, 12 (4): 817-823.

Miranda-Neto, M.J. DE. 1993. Marajó: desafio da Amazônia. Belém, CEJUP, $2^{\text {a }}$ ed., 190p.

NowAK, R.M. 1991. Walker's mammals of the world. Baltimore, Johns Hopkins Univ. Press, $5^{\text {th }}$ ed., $642 \mathrm{p}$.

1994. Walker's bats of the world. Baltimore, Johns Hopkins Univ. Press, 288p.

Overal, W.L. \& B.M. MASCARENHAS. 1993. Recomendações para o inventário faunístico da Amazônia.

Bol. Mus. Paraense Emílio Goeldi, Ser. Zool., Belém, 9 (2): 329-339.

PERES, C.A. 1989. A survey of a gallery forest primate community, Marajó Island, Pará, Brazil. Vida Silvestre Neotropical, Costa Rica, 2: 32-37.

PiCcinini, R.S. 1974. Lista provisória dos quirópteros da coleção do Museu Paraense Emílio Goeldi

(Chiroptera). Bol. Mus. Paraense Emílio Goeldi, Sér. Zool., Belém, 77: 1-32.

PIJ, L. VAN DER. 1957. The dispersal of plants by bats (Chiropterocory). Acta Botanica Neerlandica, Amsterdam, 6: 291-315.

ReIS, N.R. \& H.O.R. SCHUBART. 1979. Notas preliminares sobre os morcegos do Parque Nacional da Amazônia (Médio Tapajós). Acta Amazonica, Manaus, 9: 507-515.

Silva, M.N.F.; A.B. Rylands \& J.L. Patton. 2001. Biogeografia e conservação da mastofauna na Floresta Amazônica brasileira, p. 110-131. In: J.P.R. CAPOBIANCO; A. Veríssimo; A. Moreira; D. SAWYER; I. Dos SANTOS \& L.P. PINTO (Eds). Biodiversidade na Amazônia brasileira. São Paulo, Estação Liberdade, Inst. Socioambiental, 540p.

SiLVA JR., J.S.S. 1998. Problemas de amostragem no desenvolvimento da sistemática e biogeografia de primatas neotropicais. Neotrop. Primates, Washington, D.C., 6 (1): 21-22.

Silva JR., J.S.S. \& M.C.P. Soares. 1999. An unexpected new Record for the bush dog, Speothos venaticus Lund, 1842 in the Brazilian Amazonia (Carnivora, Canidae). Publ. Avulsas Inst. Pau Brasil Hist. Nat., São Paulo, 2: 7-11.

Simmons, N.B. \& R.S. Voss. 1998. The Mammals of Paracou, French Guiana: A Neotropical Lowland Rainforest Fauna. Part I. Bats. Bul. Amer. Mus. Nat. Hist., New York, 230: 1-219.

Slaughter, B.W. \& D.W. Walton. 1970. About Bats. Dallas: Southern Methodist University Press, $339 \mathrm{pp}$.

TADDEI, V.A. 1983, Morcegos - algumas considerações sistemáticas e biológicas. Bol. Téc. CATI Campinas, 172: 1-31.

VANZOLInI, P.E. 1970. Zoologia sistemática, geografia e a origem das espécies. Teses e Monografias, São Paulo, USP/IGEOG, 3: 1-56.

— 1977. Ecossistemas terrestres: linhas de conduta face à realidade brasileira. Biogeografia, São Paulo, USP/IGEOG, 12: 1-11.

VIVO, M. DE. 1996. How many species of mammals are there in Brazil?, p. 313-321. In: C.E. Bicudo \& N.A. Menezes (Eds.). Biodiversity in Brazil. A First Approach. Proceedings of the Workshop Methods for the Assessment of Biodiversity in Plants and Animals, Campos do Jordão, São Paulo. VizotTo, L.D. \& V.A. TAdDEI. 1973. Chave para determinação de quirópteros brasileiros. Bol. Ci. Fac. Ci. Letras São José Rio Preto 1: 1-72.

Vogel, S. 1969. Chiropterofilie in der neotropischen Flora. Neue Mitt. 3, Flora, Abt. B. 158: 289-323. Voss, R.S. \& L.H. Emmons. 1996. Mammalian Diversity In Neotropical Lowland Rainforests: A Preliminary Assessment. Bull. Amer. Mus. Nat. Hist. 230: 1-115. 
Wilson, D.E. 1989. Bats, p. 365-382 In: H. LIETH \& M.J.A. WeRgER (Eds). Tropical rainforest ecosystems: biogeographical and ecological studies. Ecosystems of the world 14B. Amsterdam, Elsevier Science Publisher, 713p.

. 1997. Bats in question. Washington, D.C., Smithsonian Inst. Press, 168p.

WILSON, D.E.; A.L. GARDNER \& R.K. LAVAL. 1970. The distributional status of some Costa Rican bats. Jour. Mammal. 51: 712-729.

WILSON, D.E. \& D.M. REEDER. 1993. Mammal species of the world: a taxonomic and geographic reference. Washington, D.C., Smithsonian Inst. Press, Amer. Soc. Mammal., $2^{\text {nd }}$ ed., 1206p.

WiLSON, D.E.; C.F. AsCORRA \& S. SOLARI. 1996. Bats as indicators of habitat disturbance, p. 613-625. In: D.E. Wilson \& A. Sandoval (Eds). Manu: The Biodiversity of Southeastern Peru. Washington, D.C., Smithsonian Inst. Press, 679p.

Recebido em 14.XII.2000; aceito em 03.IX.2002. 